\title{
Cierre percutáneo de pseudoaneurisma post-operatorio de aorta ascendente con dispositivo Amplatzer.
}

Dante Lindefjeld, Alejandro Martínez, Felipe Heusser, Ricardo Zalaquett.

Departamento de Enfermedades Cardiovasculares. Pontificia Universidad Católica de Chile. Conflictos de interés: Los autores no reportan conflictos de interés.

\section{Introducción:}

Los pseudoaneurismas de la aorta ascendente, que se desarrollan en los puntos de sutura de anastomosis entre el injerto de Dacron y la aorta, son infrecuentes, pero potencialmente fatales, y usualmente necesitan manejo quirúrgico definitivo.

Existen reportes de casos en los que se emplearon técnicas percutáneas en pacientes con riesgo quirúrgico alto. Aquí presentamos el caso de un pseudoaneurisma aórtico reparado exitosamente con el cierre percutáneo con un dispositivo Amplatzer para comunicación interventricular.

\section{Caso clínico:}

Hombre de 62 años, con antecedentes de hipertensión arterial crónica, portador de cáncer vesical, tratado y en control. Hospitalizado en noviembre de 2005 por disección aórtica tipo A de Stanford. Fue sometido a reemplazo de aorta ascendente y hemiarco aórtico con paro circulatorio, hipotermia profunda, perfusión cerebral anterógrada y canulación de la arteria axilar derecha. Se empleó la prótesis Intravascular \#28 mm, con sutura en un plano con prolene $4-0$ y 5.0 , distal y proximal respectivamente. Se conservó la válvula aórtica, hasta la unión sinutubular. La evolución fue satisfactoria. Seis meses después cursa con granuloma de la herida operatoria, que fue resecado totalmente y en el scanner de tórax se evidenció un pseudoaneurisma aórtico ascendente, proximal a la prótesis aórtica, de 45 x $29 \mathrm{~mm}$. (Figura 1,2,3)

\section{Figura 1: AngioTAC que muestra pseudoaneurisma aórtico retroesternal. Flecha blanca.}

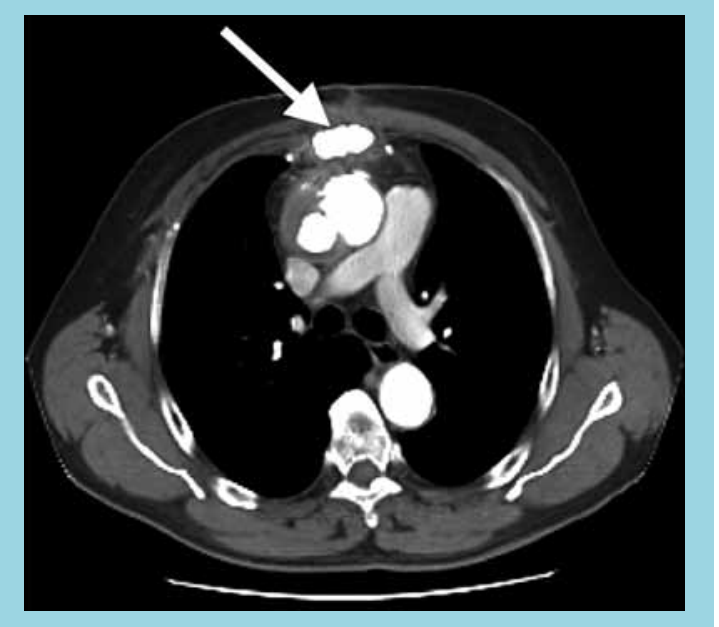

\section{Correspondencia:}

Dr. Dante S. Lindefjeld Calabi.

División de Enfermedades Cardiovasculares,

Escuela de Medicina, Pontificia Universidad Católica de

Chile, Marcoleta 367, 2do piso, Santiago - Chile.

E-mail: dslindef@puc.cl 


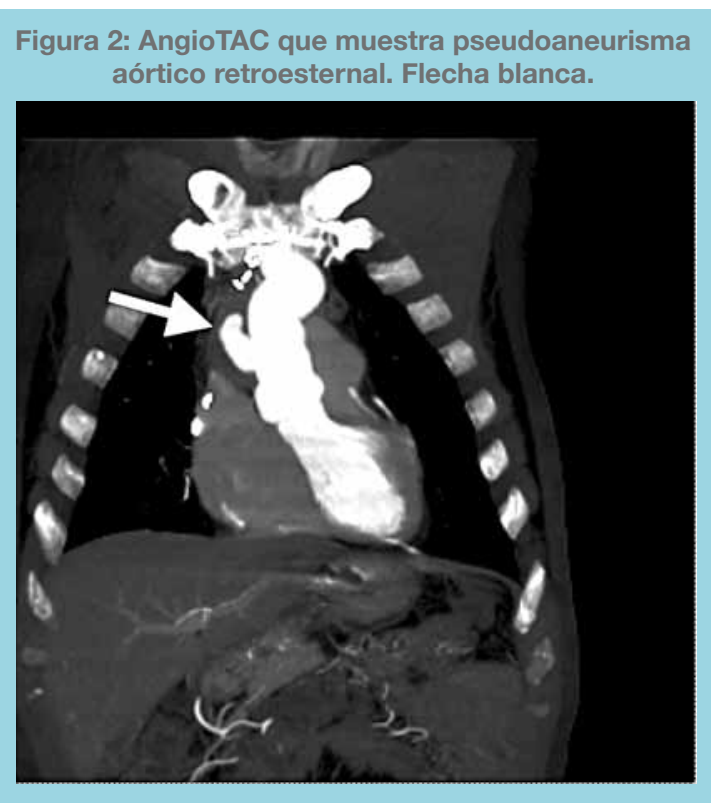

Se decide el cierre percutáneo del pseudoaneurisma. Dicho procedimiento fue efectuado el mes de agosto de 2008. Vía arterial retrógrada, se hizo la aortografía donde se visualizó el pseudoaneurisma, inyectándolo de forma selectiva con un catéter Judkins derecho \#4, 6 Fr. El aspecto era sacular, con un cuello de $9.6 \mathrm{~mm}$. (Figura 4). Debido al pobre apoyo, soporte y longitud del catéter de entrega del dispositivo Amplatzer, se seleccionó un catéter Amplatz izquierdo \#1,9 Fr. (Figura 5). Posteriormente, al canular de forma exitosa el ostium y cuello del pseudoaneurisma se liberó un Amplatzer VSD muscular de 10 mm (AGA Medical Corporation, Golden Valley, Minnesota), dejando un disco dentro del lumen del pseudoaneu-

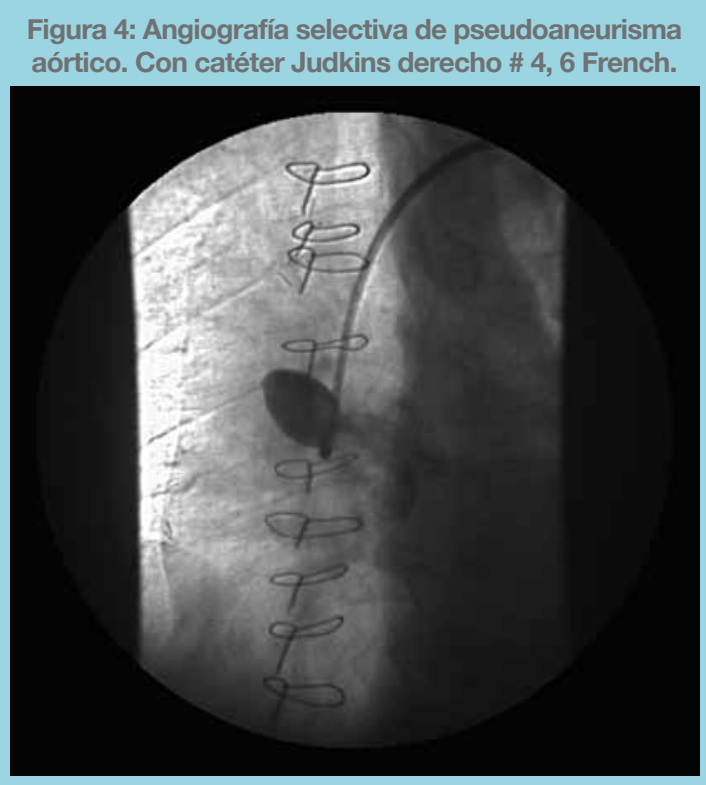

Figura 3: AngioTAC, reconstrucción con imagen de pseudoaneurisma aórtico anterior (flecha blanca), a nivel de línea anastomótica de prótesis y aaorta ascendenteretroesternal (flecha amarilla punteada).

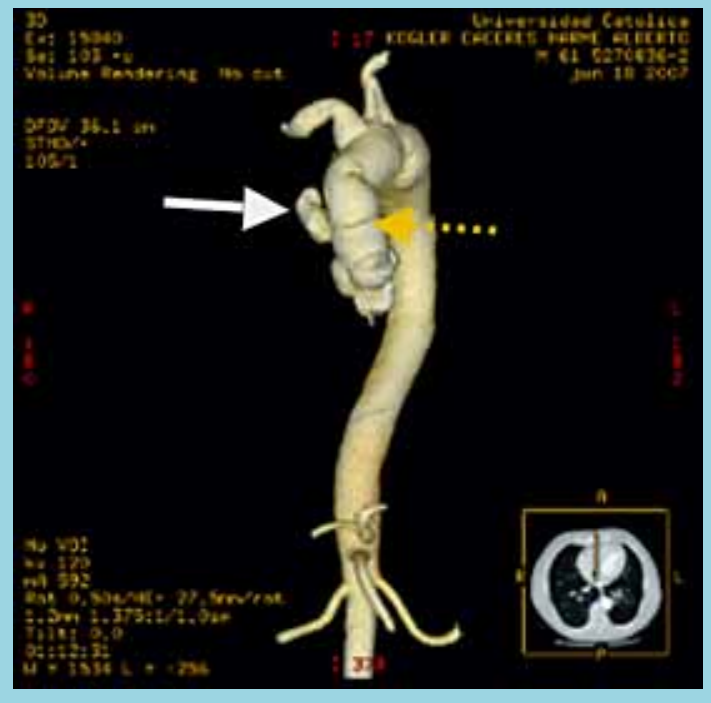

risma y el otro en la pared de la aorta.

Previo a la liberación del Amplatzer, se verificó la indemnidad del ostium de la coronaria derecha, mediante inyección selectiva a esta (Judkins \#4,6 Fr) y visualización del dispositivo (Figura 6).

Una vez implantado se observa el cierre casi total del flujo de entrada. (Figura 7)

El control radiológico y ecocardiográfico a las 24 horas confirmó posición adecuada del dispositivo. El seguimiento por imágenes alejado mostró oclusión satisfactoria del pseudoaneurisma aórtico, con buena evolución clínica en el control cardiológico cuatro años después del procedimiento,

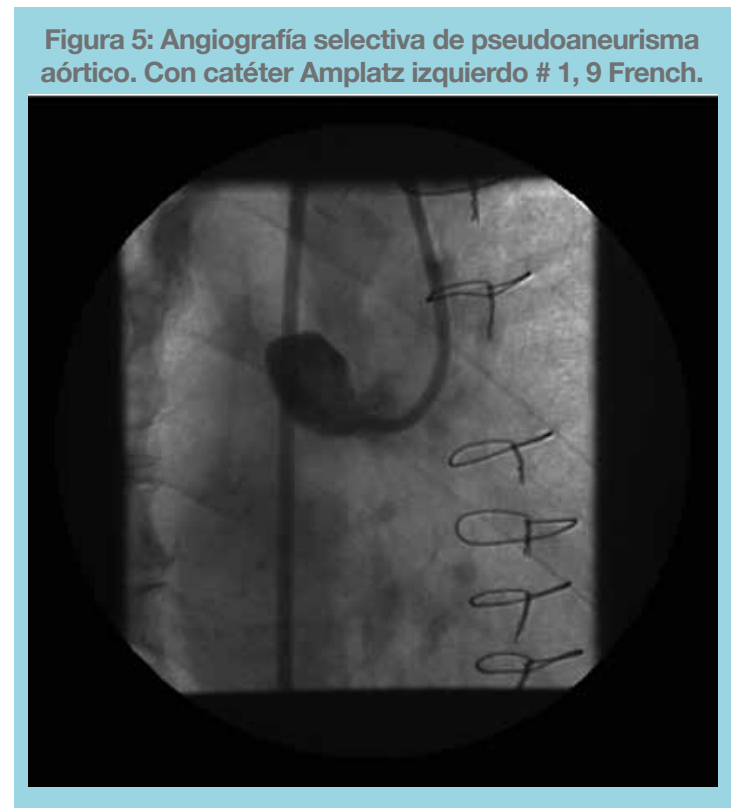




\section{Figura 6: Coronariografía selectiva coronaria derecha (flecha blanca), para descartar compromiso de su ostium con el dispositivo Amplatzer (flecha amarilla punteada).}

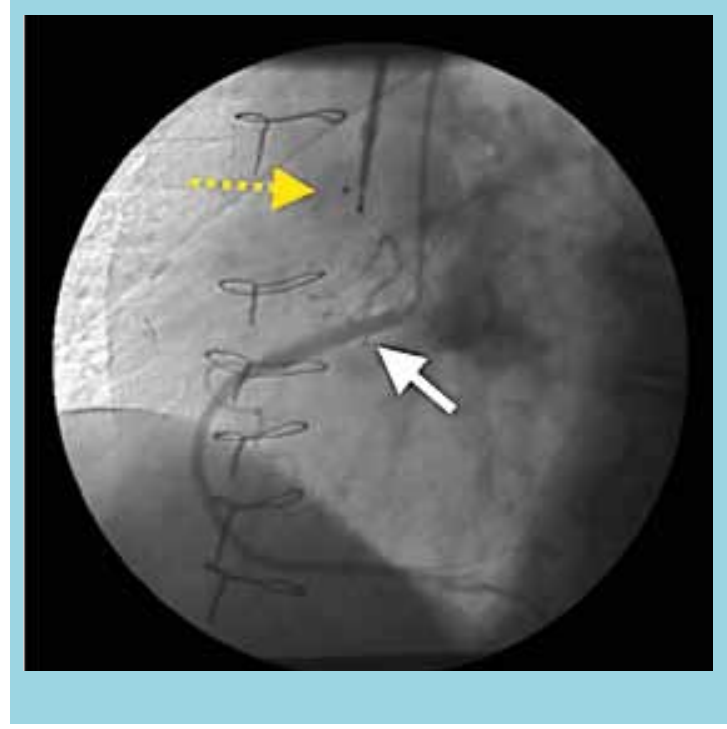

con cierre completo y sin recidiva del pseudoaneurisma aórtico.

\section{Discusión:}

Los pseudoaneurismas que se forman posteriores al reemplazo de la raíz aórtica y aorta ascendente son potencialmente fatales. La incidencia descrita oscila entre 7 a $25 \%$, siendo generalmente un hallazgo en exámenes de imágenes complementarios. Requieren habitualmente la corrección quirúrgica. ${ }^{1}$

La etiología se atribuye a la condición patológica preexistente de la aorta, alta tensión sobre la línea de sutura, infecciones, deterioro estructural de la prótesis usada y material de sutura. ${ }^{2}$

Existen reportes de casos, con técnicas percutáneas para estabilizar y facilitar la cirugía definitiva, donde se empleó la oclusión con balón (Fogarty) o depósito
Figura 7: Aortografía con catéter pigtail, que demuestra mínimo pasaje de contraste hacia el pseudoaneurisma que está ocluido con el Amplatzer, que se distingue por los puntos radio-opacos de referencia. (Flechas blancas)

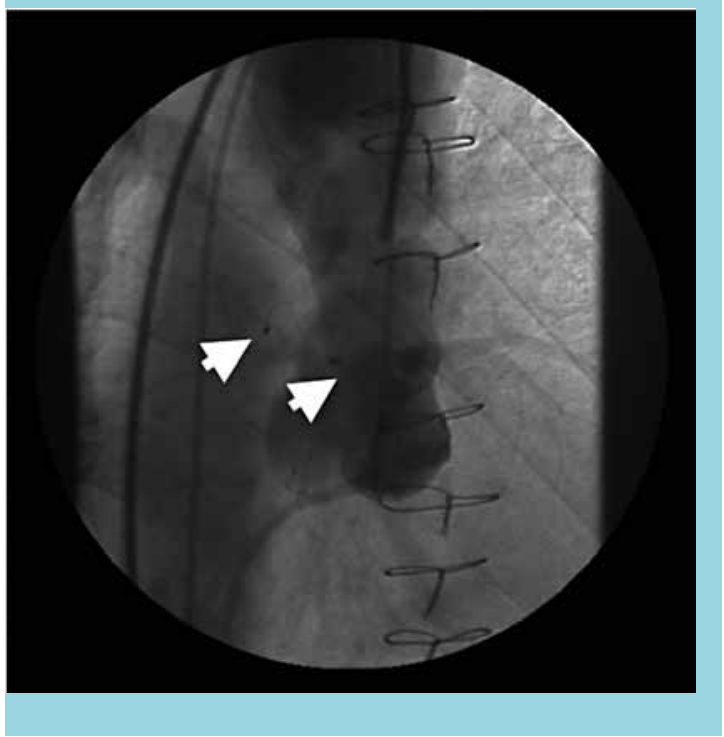

de trombina con catéter, pero con pésimos resultados por formación de trombos aórticos y embolización. ${ }^{3,4}$ También se describe el uso de coils para la embolización de los pseudoaneurismas de pacientes terminales e indicación de manejo paliativo. ${ }^{5}$

El primer cierre exitoso de un pseudoaneurisma aórtico con un dispositivo Amplatzer fue descrito en el año 2005. A partir de entonces surgen algunos reportes del cierre de este defecto con diferentes modelos de Amplatzer que fueron ajustados de acuerdo a la anatomía del pseudoaneurisma. ${ }^{6-11}$

De esta manera, aprovechando la diversidad de modelos en los dispositivos de terapia endovascular, es posible ingeniar estrategias promisorias de manejo percutáneo en la resolución de estos casos particularmente complejos. 


\section{Referencias:}

1. COSELLIJS, CRAWFORDES. Composite valve graft replacement of aortic root using separate dacron tuve for coronary artery reattachment. Ann Thorac Surg 1989; 47: 553 - 7.

2. KAYA A, SCHEPENS MA, MORSHUIS WJ, HEIJMEN RH, BRUTEL DE LA RIVIERE A, DOSSCHE KM. Valve-related events after aortic root replacement with cryopreserved aortic homografts. Ann Thorac Surg 2005; 79: 1491 - 5.

3. HATFIELD DR, FRIED AM, ELLIS GT, MATTINGLY WT JR, TODD EP. Intraoperative control of an ascending aortic pseudoaneurysm by Fogarty balloon catheter: a combined radiologic and surgical approach. Radiology 1980; 135: 515 - 7.

4. LIN PH, BUSH RL, TONG FC, CHAIKOF E, MARTIN LG, LUMSDEN AB. Intra-arterial thrombin injection of an ascending aortic pseudoaneurysm complicated by transient ischemic attack and rescued with systemic abciximab. J Vasc Surg 2001; 34: $939-42$.

5. MIGUEL B, CAMILLERI L, GABRILLARGUES J, MACHEDA B, KUBOTA H, RAVEL A, et al. Coil embolization of a false aneurysm with aorto-cutaneous fistula after prosthetic graft replacement of the ascending aorta. European Journal of Radiology 2000; 34: 57 - 59 .
6. BASHIR F, QUAIFE R, CARROLL JD. Percutaneous closure of ascending aortic pseudoaneurysm using Amplatzer septal occlude device: the first clinical case report and literature review. Catheter Cardiovasc Interv 2005; 65: 547 - 51 .

7. KOMANAPALLI CB, BURCH G, TRIPATHY U, SLATER MS, SONG HK. Percutaneous repair of an ascending aortic pseudoaneurysm with a septal occluder device. J Thorac cardiovasc Surg 2005; 130: 603 - 4 .

8. GRAHAM EM, BANDISODE VM, ATZ AM, KLINE CH, TAYLOR MH, IKONOMIDIS JS. Percutaneous occlusion of a pseudoaneurysm evolving after homograft aortic valve and root replacement with the Amplatzer muscular ventricular septal defect occlude. J Thorac Cardiovasc Surg 2006; 131: 914-6.

9. KANANI RS, NEILAN TG, PALACIOS IF, GARASIC JM. Novel use of the Amplatzer septal occlude device in the percutaneous closure of ascending aortic pseudoaneurysm: a case series. Catheter Cardiovasc Interv 2007; 69: 146 - 53.

10. SCHOLTZ W, JATEGAONKAR S, HAAS NA. Successful interventional treatment of a retrosternal pseudoaneurysm of the ascending aorta with an Amplatzer vascular Plug II. J Invasive Cardiol 2010; 22: E44 - 6.

11. ELDIEN AS, DEO S, OLUSEUN AO, RIHAL CS, JOYCE L. Envascular approach to treat aortic pseudoaneurysms: could it be a safe alternative?. Heart Surg Forum 2012; 15: E34 -6. 